\title{
ARTÍCULOS
}

\section{Estructura y crecimiento de los bosques de Araucaria angustifolia en el sur de Brasil}

\author{
Structure and growth of araucaria forest in southern Brazil
}

\author{
Emanuel Arnoni Costa ${ }^{a *}$, André Felipe Hess ${ }^{\text {a }}$, César Augusto Guimarães Finger b \\ *Autor de correspondencia: ${ }^{a}$ Universidad del Estado de Santa Catarina, Centro de Ciencias Agroveterinárias, \\ Departamento de Ingeniería Forestal, Av. Luiz de Camões, 2090, CEP 88520-000, Lages, SC, Brasil, \\ tel.: 55-49-32899255, emanuelarnonicost@gmail.com \\ ${ }^{\mathrm{b}}$ Universidad Federal de Santa Maria, Departamento de Ciencias Rurales, Rio Grande do Sul, Brasil.
}

\begin{abstract}
SUMMARY
The growth of individual trees can be simulated by multiplying a function that describes the potential by another modifier, the "modifying-potential". Modeling of the potential function for trees growing without competition determines the limit of optimum growth to species, while a modifying function sets the decrease component taking in consideration the influence of environmental factors. Thus, the aim of the study was to adjust the modifier function to describe the periodic increment in the basal area of Araucaria angustifolia trees in sites with Araucaria Forest in southern Brazil. For this, we sampled 397 individual trees. Dendrometric and morphometric variables, competition indices and increment in diameter with the chronology of widths of the growth ring were measured. Periodic increment in basal area was described by a logarithmic function that explained $57 \%$ of variance and correlated with the diameter at the beginning of the period, height, variable of crown size and competition indices. Results indicate that the modifier function can be used accurately to explain changes in growth and spatial and temporal structure of the species in the Araucaria Forest.
\end{abstract}

Key words: Parana pine, predicting growth, reductive function, forest management.

\section{RESUMEN}

El crecimiento de los árboles individuales se puede simular multiplicando una función que describe su potencial por otra modificadora "potencial-modificadora". El modelado de la función potencial para árboles creciendo sin competencia determina el límite de crecimiento óptimo para una especie, mientras que la función modificadora establece el componente de descenso teniendo en cuenta la influencia de factores ambientales. Así, el objetivo del estudio fue ajustar la función modificadora para describir el incremento periódico en el área basal de árboles de Araucaria angustifolia en sitios de bosques con araucaria en el sur de Brasil. Para eso, se tomaron muestras de 397 árboles individuales y se midieron sus variables dendrométricas, morfométricas, sus índices de competencia y su incremento en diámetro con la cronología de los anchos de anillos de crecimiento. El incremento periódico en el área basal fue descripto por una función logarítmica que explicó el $57 \%$ de la varianza y se correlaciona con el diámetro al inicio del periodo, altura, variable de dimensión de la copa y el índice de la competencia. Los resultados indican que se puede utilizar la función modificadora con precisión para explicar los cambios en el crecimiento, en la estructura espacial y temporal de la especie en el bosque de araucaria.

Palabras clave: pino brasileño, predicción del crecimiento, función reductora, manejo forestal.

\section{INTRODUCCIÓN}

Araucaria angustifolia (Bertol.) Kuntze (araucaria) es un árbol del bosque de araucaria en América del Sur. La araucaria es una especie pionera, intolerante a la sombra y domina el estrato superior del dosel. Originalmente los bosques de araucarias ocuparon $177.600 \mathrm{~km}^{2}$ en Brasil y en Argentina $2.100 \mathrm{~km}^{2}$ (Giraudo et al. 2003) en un intervalo que incluye las coordenadas de $19^{\circ} 15^{\prime}-31^{\circ} 30^{\prime} \mathrm{S}$ y $41^{\circ} 30^{\prime}-54^{\circ} 30^{\prime} \mathrm{O}$ y altitudes de 200 a $1.800 \mathrm{~m}$. En la exploración de los bosques de araucaria no se aplicaron planes de manejo sostenible y, por lo tanto, su uso fue convertido en una legislación prohibitiva, como medida de precaución y "una forma de conservación" de sus remanentes.

En este sentido, para la elaboración de planes de manejo y las intervenciones sostenidas en el bosque, son necesarios los estudios sobre el crecimiento y desarrollo estructural (espacial y temporal) de los árboles. Así, el modelado del crecimiento de los árboles puede expresarse por una función "potencial-modificadora" (Zhang et al. 2004), pues el resultado lleva en cuenta la interacción de dos factores: la multiplicación de su potencial biótico 
por un reductor que caracteriza los factores ambientales (Hasenauer 2006). La función del crecimiento potencial y modificadora representa el máximo posible en niveles de tasas de crecimiento, y los modificadores son una función del tamaño del árbol, de las condiciones del sitio, competencia y proporción de la copa (Weiskittel et al. 2011).

Por lo tanto, la importancia de los modelos de crecimiento es predecir el desarrollo de los árboles, pues es esencial para obtener informaciones consistentes, útiles al manejo de los bosques de manera racional, para la ejecución de los planes y prácticas dirigidas a las características de la especie (Costa et al. 2015). De esta manera, el ajuste de la función modificadora define un componente de descenso para el límite máximo fijado en el crecimiento potencial, proporcionando la base para la planificación de futuras intervenciones silvícolas.

El problema identificado es que la mayoría de los estudios de modelos de crecimiento para araucaria no tienen en cuenta las variables que caracterizan la calidad del sitio ni el efecto de la competencia. Así, se buscó agregar esas variables en los modelos para comprobar la influencia de estos elementos en el comportamiento biológico, en la densidad y en la estructura del bosque, con el objetivo de modelar una "función modificadora" asociada con variables dendrométricas, del sitio y de densidad (competencia), para representar el componente de descenso de las tasas del incremento periódico en área basal de la especie. En el modelado de la función modificadora de los árboles objetivo, se supone que el incremento periódico anual en área basal es una función del tamaño actual, la historia de vigor y la competencia actual.

Por lo anterior, se proponen las preguntas: 1) ¿cuáles variables explican mejor la relación con el incremento periódico en el área basal y cuál es el valor de su coeficiente de correlación? y 2) ¿el modelado de la función modificadora define un componente de descenso del crecimiento para el límite máximo establecido en potencial de crecimiento? Se plantea la hipótesis que cuando a los modelos de crecimiento se incorporan las variables de sitio, competencia y morfométricas, estas mejoran su desempeño y explican el desarrollo en la estructura individual de los árboles de araucaria. Por lo tanto, el objetivo de este estudio es modelar una ecuación modificadora para describir el incremento periódico en área basal de árboles de araucaria.

\section{MÉTODOS}

Área de estudio. El estudio se realizó en dos sitios con bosque de araucaria en el sur de Brasil: Lages (LAG), en Santa Catarina, y São Francisco de Paula (SFP), en Rio Grande do Sul. El clima de los municipios, según la clasificación de Köppen, es subtropical húmedo, sin estación seca, el verano templado (Cfb) (Alvares et al. 2013) y con las siguientes características climáticas (cuadro 1):

El inventario fitosociológico en los dos sitios de estudio identificó a la especie araucaria como la de mayor valor de importancia (VI), siendo su valor el $28 \%$ en LAG y el 36 $\%$ en SFP. En LAG, se identificaron a 28 especies y 10 especies representan el 82,9\% del total; en SFP, 10 especies corresponden a un $71,4 \%$ del total. En LAG, el estudio se realizó en un bosque nativo de dominio privado, con densidad de 303 árboles por hectárea; en SFP, en un área de bosque nacional (FLONA) con densidad de 220 árboles por hectárea, establecida por el gobierno federal de Brasil.

Toma de datos. Fueron muestreados 397 árboles en un enfoque basado en árbol individual en la amplitud de distribución de diámetro en los dos sitios. Todos los árboles a partir de $10 \mathrm{~cm}$ de diámetro a la altura del pecho se midieron y se consideró una amplitud entre las clases de $10 \mathrm{~cm}$. Los árboles de araucaria en competencia fueron llamados "árboles objetivo" y los que ejercen la competencia "competidores". El criterio de selección de árboles "competidores" en relación con el árbol "objetivo" fue basado en la dimensión de la copa, y la altura de los árboles, en la capacidad de competir por la luz y el espacio de crecimiento. Así, todos los árboles en un radio de $360^{\circ}$ del árbol objetivo y que tuvieron contacto entre las copas fueron considerados competidores, y su criterio de selección se obtuvo con la ecuación 1 .

$$
\operatorname{dist}_{\mathrm{ij}}<\left(\mathrm{rc}_{\mathrm{i}}+\mathrm{rc}_{\mathrm{j}}\right)
$$

Donde: dist $t_{\mathrm{ij}}$ distancia horizontal entre el árbol objetivo (i) y el árbol competidor (j); $\mathrm{rc}_{\mathrm{i}}, \mathrm{rc}_{\mathrm{j}}$ : radio de la copa de los árboles objetivo (i) y competidor (j) en metros.

El radio de la copa (rc) fue calculado dividiendo por dos el valor obtenido de la estimación del diámetro de la copa $(\mathrm{dc})$ como una función del diámetro a la altura del pecho (DAP),

Cuadro 1. Coordenadas geográficas y características climáticas de los sitios de estudio.

Geographic coordinates and climatic characteristics of the study sites.

\begin{tabular}{lccccc}
\hline \multicolumn{1}{c}{ Municipios } & Latitud S & Longitud O & Altitud (m) & TMA & PMA \\
\hline Lages (LAG) & $-27^{\circ} 48^{\prime}$ & $-50^{\circ} 19^{\prime}$ & 987,0 & 15,2 & $1.684,7$ \\
São Francisco de Paula (SFP) & $-29^{\circ} 26^{\prime}$ & $-50^{\circ} 35^{\prime}$ & 854,0 & 15,0 & $2.016,4$ \\
\hline
\end{tabular}

$\mathrm{TMA}=$ temperatura media anual en ${ }^{\circ} \mathrm{C} ; \mathrm{PMA}=$ precipitación media anual en milímetros. 
con ecuaciones para cada sitio. Para LAG: $[\mathrm{dc}=1,3149+$ 0,2112 . DAP], con coeficiente de determinación $\left(\mathrm{R}^{2}\right)=0,838$ y error estándar de estimación $\left(\mathrm{S}_{\mathrm{yx}} \%\right)$ de 13,7 y SFP: [dc = $0,8947+0,2032$. DAP], con $\left(\mathrm{R}^{2}\right)=0,853$ y $\left(\mathrm{S}_{\mathrm{yx}} \%\right)=15,4$.

De todos los árboles (objetivo y competidor) se midieron el diámetro a la altura del pecho (DAP), altura total (h), altura de inserción de la copa (hic) y ocho radios de la copa en las direcciones cardinales. Con los datos, se calcularon las variables morfométricas para araucaria, en los dos sitios de estudio (cuadro 2), y ocho índices de competencias independientes y dependientes de la distancia.
Cronologías de ancho de anillos. Para cada árbol en cada sitio, se tomaron dos tarugos de incremento en el diámetro a la altura del pecho, con barreno de Pressler. Los tarugos de incremento fueron pegados en soportes de madera, lijados y marcados sus anillos por un período de cinco años. Se escanearon las muestras y se almacenaron las imágenes a una resolución de 1.200 píxeles. Con la digitalización de las muestras, se midieron los incrementos con el software Imagen Pro-Plus, con escala en milímetros, y se hizo su registro en archivo de texto. El incremento periódico anual en área basal se obtuvo con la ecuación 2.

Cuadro 2. Características dendrométricas, morfométricas e índices de competencia utilizados para los dos sitios de estudio de Araucaria angustifolia en el sur de Brasil.

Dendrometric characteristics, morphometric and competition indices used for the two study sites of Araucaria angustifolia in southern Brazil.

\begin{tabular}{|c|c|c|c|c|c|}
\hline Variable & Mínimo & Media & & Máximo & $\mathrm{CV} \%$ \\
\hline DAP & 9,9 & 40,2 & & 93,0 & 43,8 \\
\hline $\mathrm{h}$ & 7,2 & 17,3 & & 25,1 & 21,8 \\
\hline hic & 3,0 & 13,1 & & 22,3 & 30,1 \\
\hline $\mathrm{dc}$ & 2,2 & 9,5 & & 22,6 & 40,9 \\
\hline APHC & 3,8 & 82,6 & & 401,1 & 78,9 \\
\hline $\mathrm{Pc}$ & 5,1 & 25,6 & & 75,0 & 50,4 \\
\hline $\mathrm{cc}$ & 0,6 & 4,2 & & 10,7 & 43,4 \\
\hline $\mathrm{Fc}$ & 0,61 & 2,54 & & 6,70 & 48,8 \\
\hline $\mathrm{h} / \mathrm{DAP}$ & 22,7 & 49,0 & & 108,8 & 33,9 \\
\hline IA & 0,16 & 0,54 & & 1,15 & 31,3 \\
\hline IS & 0,12 & 0,24 & & 0,38 & 18,1 \\
\hline IC & Expresión & & Variable & \multicolumn{2}{|c|}{ Fuente } \\
\hline $\mathrm{IC} 1$ & $=D A P_{i}^{2} \cdot n /\left(\sum_{j=1}^{n} D A P_{j}^{2}\right)$ & & DAP & \multicolumn{2}{|c|}{ Daniels et al. (1986) } \\
\hline $\mathrm{IC} 2$ & $=\sum_{j=1}^{n}\left(D A P_{j}^{2} / D A P_{i}^{2}\right)$ & & DAP & \multicolumn{2}{|c|}{ Corona y Ferrara (1989) } \\
\hline IC3 & $=\left(\sum_{j=1}^{n} D A P_{j}\right) / D A P_{i}$ & & DAP & \multicolumn{2}{|c|}{ Lorimer (1983) } \\
\hline $\mathrm{IC} 4$ & $=\left(\sum_{j=1}^{n} D A P_{j} / D A P_{i}\right) / n$ & & DAP & \multicolumn{2}{|c|}{ Mugasha (1989) } \\
\hline IC5 & $=\sum_{j=1}^{n}\left[D A P_{j} /\left(D A P_{i} \cdot\right.\right.$ distij $\left.)\right]$ & & DAP, dist & \multicolumn{2}{|c|}{ Hegyi (1974) } \\
\hline IC6 & $=\sum_{j=1}^{n}\left[D A P_{j} /\left(D_{A} P_{i} \cdot \operatorname{distij}^{0,5}\right)\right]$ & & DAP, dist & \multicolumn{2}{|c|}{ Hegyi (1974) - modificado } \\
\hline IC7 & $=\sum_{j=1}^{n}\left[\left(D A P_{j} / D A P_{i}\right)^{2} /\right.$ distij $]$ & & DAP, dist & \multicolumn{2}{|c|}{ Rouvinen y Kuuluvainen (1997) } \\
\hline IC8 & $=\sum_{j=1}^{n}\left(D A P_{j} /\right.$ distij $)$ & & DAP, dist & \multicolumn{2}{|c|}{ Rouvinen y Kuuluvainen (1997) } \\
\hline
\end{tabular}

DAP: diámetro a la altura del pecho en cm; h: altura total en m; hic: altura de inserción de la copa en m; dc: diámetro de la copa en m; APHC: área de proyección horizontal de la copa en $\mathrm{m}^{2}$; Pc: proporción de la copa en \%; cc: longitud de la copa en m; Fc: formal de copa; h/DAP: grado de esbeltez; IA: índice de amplitud; IS: índice saliente; CV\% coeficiente de variación; IC1...IC8: índices de competencia; dist: distancia horizontal en m; DAP: diámetro del árbol objetivo; $\mathrm{DAP}_{\mathrm{j}}$ : diámetro del árbol competidor. 


$$
\operatorname{IPAg}=\left(\mathrm{g}-\mathrm{g}_{5}\right) / \mathrm{t}
$$

Donde: IPAg: incremento periódico anual en área basal $\left(\mathrm{cm}^{2}\right.$ año $\left.^{-1}\right)$; g: área basal del árbol individual al final del período $\left(\mathrm{cm}^{2}\right)$; $\mathrm{g}_{5}$ : área basal del árbol individual al inicio del período $\left(\mathrm{cm}^{2}\right)$; t: número de años evaluados.

Modelado del incremento periódico anual en área basal. Se utilizaron el análisis de correlación de Pearson, para indicar el grado de asociación entre las variables independientes para modelar el incremento periódico anual en área basal, y el procedimiento stepwise, para determinar las variables con mayor influencia y que pueden explicar los cambios estructurales y el crecimiento de los árboles de araucaria en su área de distribución natural. Para esta relación, se utilizó la ecuación 3, propuesta por Wykoff (1990).

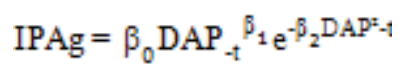

Donde: IPAg: incremento periódico anual en área basal $\left(\mathrm{cm}^{2}\right.$ año $\left.{ }^{-1}\right)$; DAP-t: diámetro a la altura del pecho al inicio del período en centímetros; $\mathrm{DAP}^{2}$-t: diámetro a la altura del pecho al cuadrado al inicio del período en centímetros, y que representa el componente de descenso, lo que provoca aumento o disminución e impide el crecimiento ilimitado para los árboles de gran diámetro; $\beta$ 's: coeficientes de regresión; e: base del logaritmo natural.

El histórico de vigor de los árboles objetivo fue valorado mediante la multiplicación de la ecuación 3 por exponenciales de las variables que caracterizan las dimensiones de la copa, siendo considerado el primer modificador del crecimiento (ecuación 4).

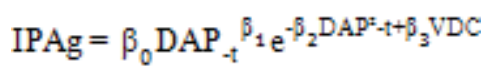

Donde: IPAg: incremento periódico anual en área basal $\left(\mathrm{cm}^{2}\right.$ año $\left.{ }^{-1}\right)$; DAP-t: diámetro a la altura del pecho al inicio del período en centímetros; $\mathrm{DAP}^{2}$-t: diámetro a la altura del pecho al cuadrado al inicio del período en centímetros; VDC: variable de la dimensión de la copa; $\beta$ 's: coeficientes de regresión; e: base del logaritmo natural.

La intensidad de la competencia actual del árbol objetivo por sus competidores se evaluó mediante la multiplicación de la ecuación 4 por la exponencial del índice de competencia, siendo considerado el segundo modificador del crecimiento (ecuación 5).

$$
\text { IPAg }=\beta_{0} D_{-1} P_{-t} \beta_{1} e^{-\beta_{2} D A P^{2}-t+\beta_{3} V D C-\beta_{4} I C}
$$

Donde: IPAg: incremento periódico anual en área basal $\left(\mathrm{cm}^{2}\right.$ año $\left.{ }^{-1}\right)$; DAP-t: diámetro a la altura del pecho al inicio del período en centímetros; $\mathrm{DAP}^{2}$-t: diámetro a la altura del pecho al cuadrado al inicio del período en centímetros; VDC: variable de la dimensión de la copa; IC: índice de competencia; $\beta$ ’s: coeficientes de regresión; e: base del logaritmo natural.

Análisis de los datos. Los análisis estadísticos se realizaron en el Sistema de Análisis Estadístico (SAS 2004). La precisión de ajuste de los modelos se evaluó mediante el coeficiente de determinación $\left(\mathrm{R}^{2}\right)$, error estándar de la estimación $\left(\mathrm{S}_{\mathrm{yx}}\right)$ y análisis gráfico de los residuos. Se linealizó la ecuación 5, y las variables independientes incluidas en el modelo obtuvieron factor de inflación de varianza (FIV) menor que 10 y la transformación propuesta por Box y Cox (1964), con valor de lambda $(\lambda=0)$, indicó la transformación logarítmica adecuada para reducir la varianza de la variable dependiente (figura 1). Esta transformación en el modelado de crecimiento puede mejorar la estructura residual y reducir la heterogeneidad de los residuos del modelo. Debido a la discrepancia logarítmica, se volvió a calcular el error estándar de la estimación $\left(\mathrm{S}_{\mathrm{yx}}\right)$ multiplicando su valor por el factor de corrección (Fcor. $=e^{0,55 y x^{2}}$ ), propuesto por Sprugel (1983).

\section{RESULTADOS}

Modelado del incremento periódico en área basal. Los cambios estructurales y de crecimiento de la especie indicaron correlación entre el incremento periódico anual en área basal (IPAg) y las variables descriptivas del diámetro al inicio del periodo (DAP-t), la altura (h), la historia de vigor ( $\mathrm{Pc} \%$ : proporción de la copa) y el índice de competencia (IC3), todas con valor de $(P<0,0001)$ (figura 2$)$.

La correlación mostró que el aumento en el diámetro y la altura que describe el tamaño actual, así como la proporción de la copa, promovieron el aumento en el incremento periódico anual en área basal, lo que indica que los árboles con copas cortas y anchas tienen menor aumento en comparación con los árboles de copas largas y estrechas, que son más eficientes en el aumento de las tasas de incremento. Los resultados muestran también que la competencia provocó reducción en las tasas de incremento en área basal (figura 3).

El procedimiento stepwise, para modelar el incremento periódico anual en área basal, fue compuesto por las variables DAP en el inicio del período y la altura $(30,37 \%)$, la variable proporción de la copa $(24,2 \%)$ y el índice de la competencia $(2,85 \%)$, que en conjunto explicaron $\left(\mathrm{R}^{2}\right)$ el $57,42 \%$ de la variación del incremento, el valor del error estándar de la estimación $\left(\mathrm{S}_{\mathrm{yx}}\right)$ de 0,46 y todos los coeficientes del modelo con valor de $(P<0,001)$. Por lo tanto, el incremento periódico anual en área basal de árboles individuales y sus cambios estructurales y el crecimiento en el bosque puede ser predicho con precisión por la ecuación 6 , con sus respectivos coeficientes.

IPAg $=0,339963$ DAP $_{-t}^{0.8799761} \mathrm{e}^{-0.000084 \mathrm{DAP} \mathrm{P}^{2}+t+0.032871 \mathrm{~h}+0.025326 \mathrm{PC}-0.06669101 \mathrm{C} 3}[6]$

Donde: IPAg: incremento periódico anual en área basal en $\mathrm{cm}^{2}$ año ${ }^{-1}$; DAP-t: diámetro a la altura del pecho al inicio 

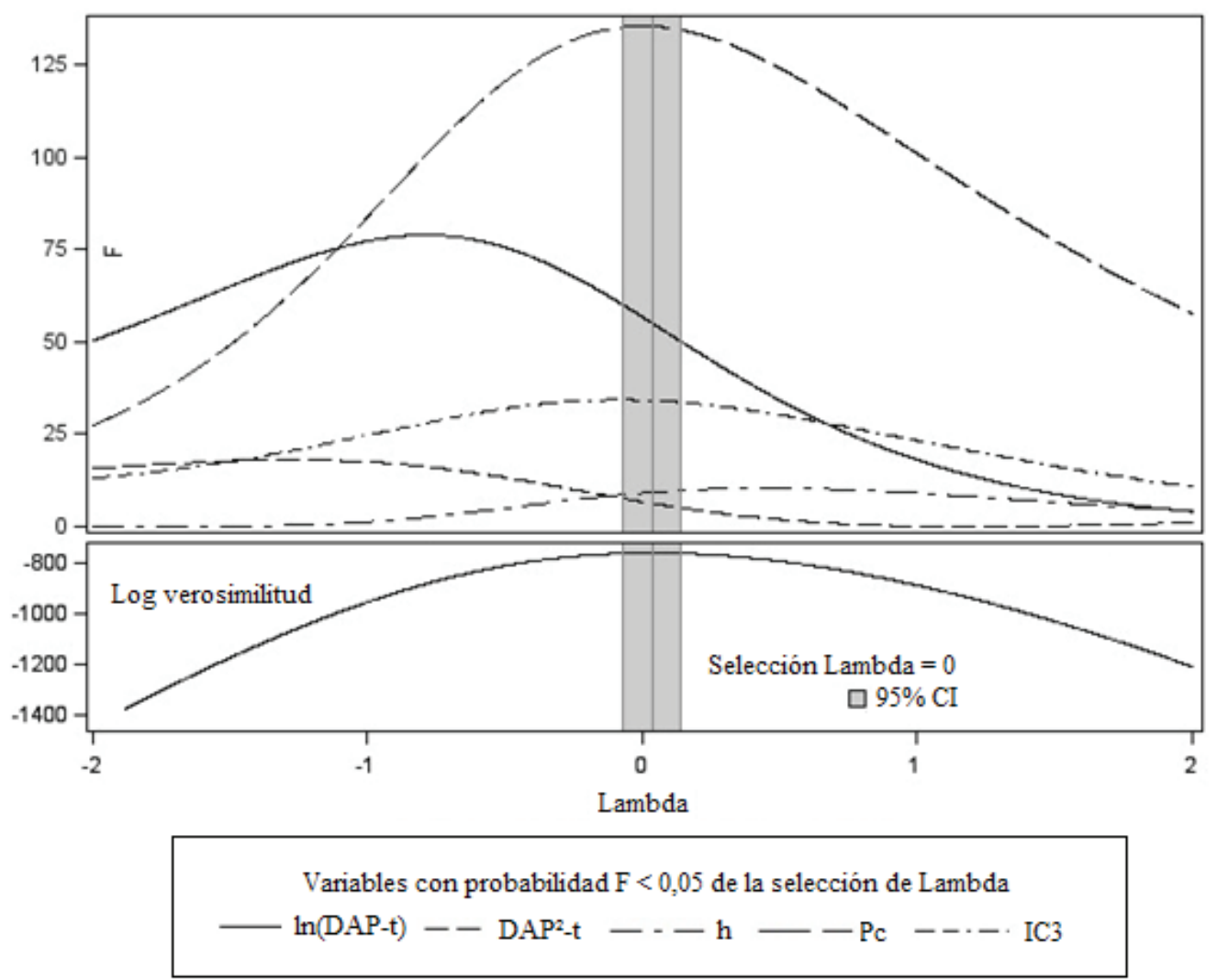

Figura 1. Variables con probabilidad $\mathrm{F}<0,05$ y el perfil de verosimilitud de la selección de valor de lambda para describir el incremento periódico en área basal de árboles muestreados en los sitios de estudio en el sur de Brasil.

Variables with probability $\mathrm{F}<0.05$ and likelihood profile of the lambda value selection to describe the periodic increment in basal area of trees sampled in the study sites in southern Brazil.

del período en centímetros; $\mathrm{DAP}^{2}$-t: diámetro a la altura del pecho al cuadrado al inicio del período en centímetros; h: altura en metros; Pc\%: proporción de la copa; IC3: índice de competencia; e: base del logaritmo natural.

La competencia ejercida por otros árboles en el árbol objetivo, cuantificado por el índice de competencia (figura 3E), influyó en la morfometría, especialmente, en las variables asociadas con el tamaño de la copa. El análisis de los residuos en función del DAP al comienzo del período (DAP-t) (figura 3F) mostró una distribución homogénea sin la presencia de errores sistemáticos que sobrestiman o subestiman valores.

\section{DISCUSIÓN}

La función y sus correlaciones. La función modificadora ajustada presenta uso potencial para la construcción de modelos cuando se quiere lograr una mayor precisión de las estimaciones. Los resultados muestran la dependencia de la relación del incremento periódico anual en área basal con el diámetro al inicio del período y las variables de la copa, lo que indica que el crecimiento y la dinámica es- tructural del bosque se ven afectados por los cambios en la forma de los árboles.

La correlación positiva del incremento periódico con esas variables muestra que el aumento de las tasas de incremento es proporcional al aumento de las variables y se puede utilizar como una relación de proximidad entre la asignación de recursos y el modelado de la dinámica del bosque. Pretzsch y Dieler (2011) demostraron con sus estudios que el modo de la competencia y las relaciones interdimensionales pueden cambiar con los gradientes ecológicos y, temporalmente, con el desarrollo del bosque.

La correlación entre el incremento y las variables de diámetro y de dimensión de copa muestra su importancia para indicar la capacidad de producción del bosque, pues cuanto mayor la dimensión de copa, mayor el área de copa, mejor su potencial fotosintético y mayor el incremento diamétrico. Para eso, los árboles necesitan mayor extensión de la copa y, por consiguiente, necesidad de espacio para su expansión y de recursos bióticos y abióticos (Lang et al. 2010) para su desarrollo. Además, con sus cambios estructurales (cambios en la forma con el tiempo, ingreso de nuevos individuos, sucesión forestal) se puede planificar la necesidad de las futuras intervenciones silvícolas. 

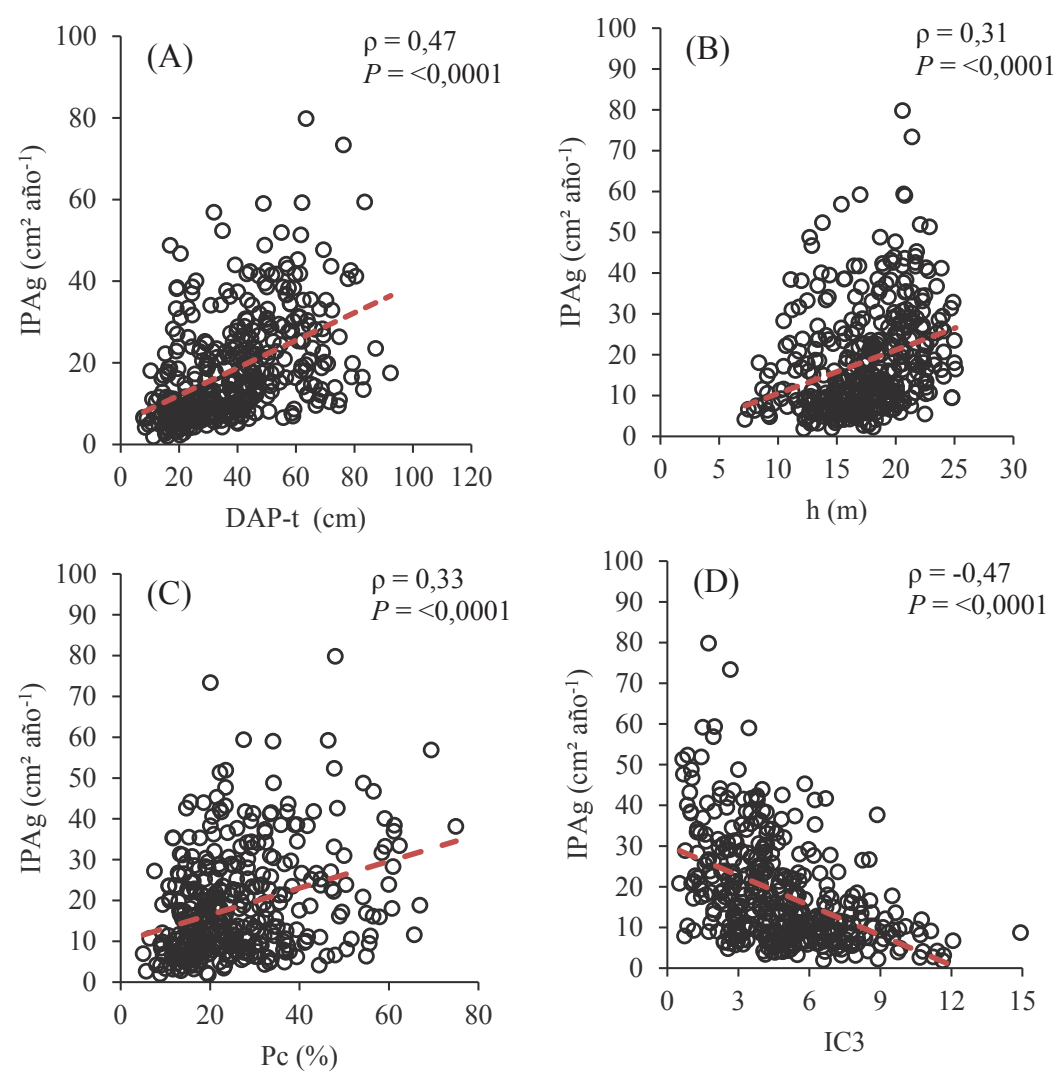

Figura 2. Correlación de Pearson ( $\rho$ ) y análisis gráfico del incremento periódico anual en área basal (IPAg) en función de las variables: A) DAP-t: diámetro a la altura del pecho al inicio del período en centímetros; B) altura (h) en metros; C) proporción de la copa (Pc\%); D) índice de competencia (IC3); de árboles muestreados en los sitios de estudio en el sur de Brasil.

Pearson correlation $(\rho)$ and graphical analysis of the annual periodic increment in basal area (IPAg) as a function of variables: A) DAP-t: diameter at breast height at the beginning of the period in $\mathrm{cm}$; B) height in $\mathrm{m}(\mathrm{h})$; C) proportion of the crown (Pc\%); D) competition index (IC3); of the trees sampled in the study sites in southern Brazil.

Los resultados muestran la lógica de esta relación, pues el incremento en el área basal mostró una correlación negativa con el índice de competencia, o sea, el incremento y el crecimiento de los árboles tienen relación directamente proporcional con el tamaño de la copa, del espacio y con los recursos del sitio, e inversamente proporcional con la competencia, que afecta a la expansión, el crecimiento y la dinámica estructural del bosque. Los resultados son consistentes con la investigación realizada para el modelado de los árboles individuales de otras coníferas (Monserud y Sterba 1996).

De este modo, las relaciones interdimensionales de los árboles pueden predecir el espacio requerido para el árbol en todo su desarrollo, juzgando así su competencia y permitiendo llegar a conclusiones acerca de la estabilidad, la vitalidad y la productividad de cada individuo, que sirve como una herramienta práctica para intervenciones silvícolas, especialmente, cuando no se conoce la edad de los árboles (Durlo 2001).

Incremento periódico y cambios estructurales. El índice de competencia (IC3) representa en la función modificadora el componente de descenso del incremento periódico anual en área basal, ya que mide las condiciones actuales de la competencia, mientras que los índices morfométricos en la función expresan el efecto de la competencia en el pasado. Eso explica los cambios estructurales y en la dinámica de las copas como factores que reducen la capacidad de crecimiento de los árboles. Cole y Lorimer (1994) obtuvieron aumento en el ajuste de las estimaciones de incremento en área basal cuando incluyeron en los modelos variables de área de proyección de la copa, porcentaje de área de la copa expuesta y la altura relativa, con valores de coeficiente de determinación de 0,77 a 0,88 y un aumento del 13 al $47 \%$ en la precisión del ajuste, en comparación con ecuaciones con solamente variables de competencia en función del diámetro a la altura del pecho para Acer saccharum Marshall, Fraxinus americana L. y Tilia americana L.

Los resultados revelan que el desarrollo de las variables de forma de los árboles, acompañado con los cambios en la estructura del bosque, afectan a lo largo del tiempo los procesos tales como la absorción de luz, la intercepción y evapotranspiración del agua, la fotosíntesis y la respira- 
ción, que a su vez influye en el crecimiento de los árboles y en el ciclo de vida de los organismos (Pretzsch 2009).

Según el mismo autor, la estructura de los bosques determina la competencia por los recursos, la producción de biomasa y las condiciones de crecimiento de las especies, así como la renovación del sistema y el desarrollo de la regeneración. La estructura de la copa es crucial para el ciclo de retroalimentación de los árboles entre la funcionalidad y la estructura en el medio ambiente, principalmente en bosques mixtos, donde diferentes especies muestran su
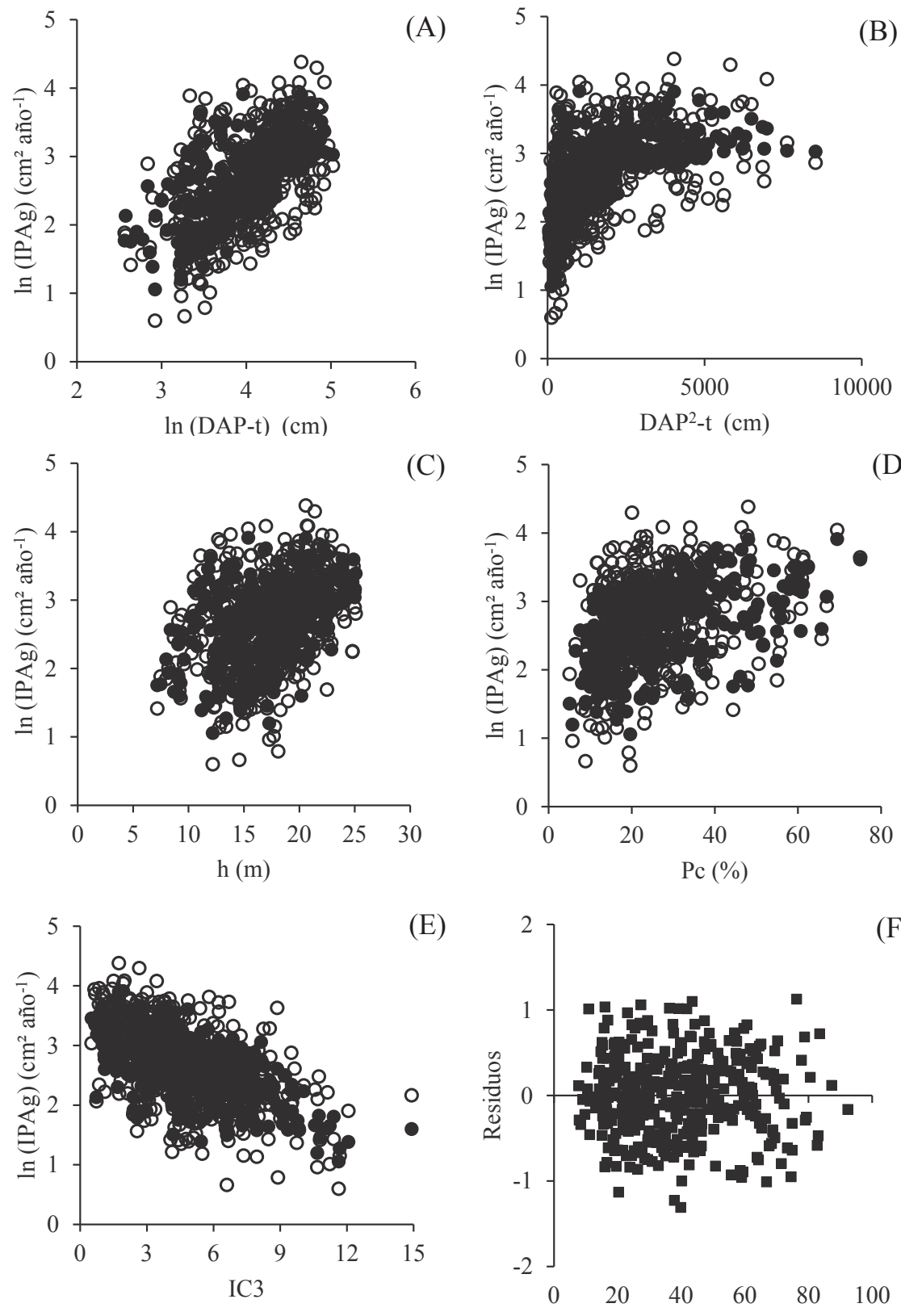

(E)

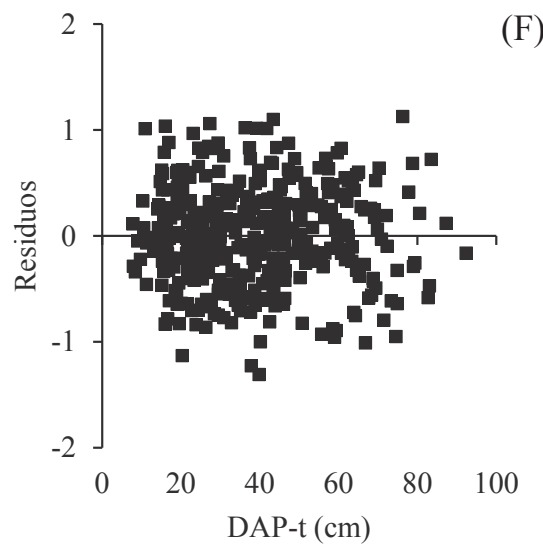

(F)

Figura 3. Valores observados y predichos de la ecuación de ajuste de incremento periódico en el área basal en función de cada variable independiente: A) ln (DAP-t): logaritmo del diámetro a la altura del pecho al inicio del período en centímetros; B) DAP²-t: diámetro a la altura del pecho al cuadrado al inicio del período en centímetros; C) h: altura en metros; D) Pc\%: proporción de la copa; E) IC3: índice de competencia; F) residuos en función del diámetro a la altura del pecho al inicio del periodo en centímetros.

Observed and estimated values of the adjusted equation of periodic increment in basal area as a function of each independent variable: A) ln (DAP-t): logarithm of diameter at breast height at the beginning of the period in $\mathrm{cm}$; B) DAP²-t: diameter at breast height at the beginning of the period squared in $\mathrm{cm}$; C) h: height in $\mathrm{m}$; D) Pc \%: proportion of the crown; E) IC3: competition index; F) residuals in function of diameter at breast height at the beginning of the period in $\mathrm{cm}$. 
capacidad para adaptar su estructura, capturar los recursos escasos eficientemente o denegar el acceso a los competidores por los mismos recursos.

El descenso del incremento en el área basal se comprueba cuando se produce un aumento en la densidad de árboles en los bosques y muestra la eficiencia del modelo para evaluar la dinámica del bosque y de su aplicación en el manejo forestal. Lo que justifica el uso de raleo selectivo sostenible, que contribuya a la reanudación del crecimiento y de la regeneración natural (Souza et al 2008), porque actualmente, por no sufrir intervenciones silvícolas, los remanentes de Bosques de Araucaria presentan tasas mínimas de regeneración.

\section{CONCLUSIONES}

La función modificadora mostró una mejoría en la capacidad de predicción, que tiene como variables actuales de tamaño, el diámetro a la altura del pecho y la altura total del árbol. Por lo tanto, la variable proporción de la copa expresa la competencia pasada y la variable índice de competencia es un descriptor de la competencia actual.

La función modificadora explica los cambios en la estructura y la dinámica del bosque, mostrando que esos cambios se producen hasta un punto máximo, en el que la densidad y la competencia indican el descenso del crecimiento y la necesidad de intervenciones silvícolas.

La reducción del incremento con el aumento de la densidad y competencia afecta a la formación de árboles con mayor diámetro en el futuro, debido al estancamiento o estabilización del crecimiento, lo que ocasiona cambios en estructura y dinámica de diámetros del bosque, compromete la reanudación de su crecimiento (mismo con intervenciones) y afecta a la expansión de su copa.

\section{REFERENCIAS}

Alvares CA, JL Stape, PC Sentelhas, JLM Gonçalves, G Sparovek. 2013. Köppen's climate classification map for Brazil. Meteorologische Zeitschrift 22(6): 711-728.

Box GEP, DR Cox. 1964. An analysis of transformations. Journal of the Royal Statistical Society 26(2): 211-243.

Cole WG, CG Lorimer. 1994. Predicting tree growth from crown variables in managed Northern hardwood stands. Forest Ecology and Management 67: 159-175.

Corona P, A Ferrara. 1989. Individual competition indices for conifer plantations. Agriculture, Ecosystems \& Environment 27: 429-437.

Costa EA, CAG Finger, AF Hess. 2015. Modelo de incremento em área basal para árvores de araucária de uma floresta inequiânea. Pesquisa Florestal Brasileira 35(83): 239-245.

Daniels RF, HE Burkhart, TR Clason. 1986. Comparison of competition measures for predicting growth of loblolly pine trees. Canadian Journal for Forest Research 16(2): 1230-1237.

Durlo MA. 2001. Relações morfométricas para Cabralea canje- rana (Well.) Mart. Ciência Florestal 11(1): 141-150.

Giraudo AR, H Povedano, MJ Belgrano, E Krauczuk, U Pardiñas, A Miquelarena, D Ligier, D Baldo, M Castelino. 2003. Biodiversity status of the interior Atlantic forest of Argentina. In Galindo-Leal C, V Câmara eds. The Atlantic Forest of South America: Biodiversity Status, Threats, and Outlook. Washington DC, USA. Island Press. p. 160 - 180.

Hasenauer, H. 2006. Sustainable Forest Management: Growth Models for Europe. Berlin, Germany. Springer-Verlag. 398 p.

Hegyi FA. 1974. A simulation model for managing jack-pine stands. In Fries J ed. Growth models for tree and stand simulation. Stockolm, Sweden. Royal College of Forestry 30. p. 74-90.

Lang AC, W Härdtle, H Bruelheide, C Geibler, K Nadrowski, A Schuldt, M Yu, G von Oheimb. 2010. Tree morphology responds to neighbourhood competition and slope in species-rich forests of subtropical China. Forest Ecology and Management 260: 1708-1715.

Lorimer CG. 1983. Tests of age independent competition indices for individual trees in natural hardwood stands. Forest Ecology and Management 6: 343-360.

Monserud RA, H Sterba. 1996. A basal area increment model for individual trees growing in even-and-uneven-aged forest stands in Austria. Forest Ecology and Management 80: 57-80.

Mugasha AG. 1989. Evaluation of Simple Competition Indices for the Prediction of Volume Increment of Young Jack Pine and Trembling Aspen Trees. Forest Ecology and Management 26: 227-235.

Pretzsch H. 2009. Forest dynamics, growth and yield. From measurement to model. Berlin, Germany. Springer. 664 p.

Pretzsch H, J Dieler. 2011. The dependency of the size-growth relationship of Norway spruce (Picea abies [L.] Karst.) and European beech (Fagus sylvatica [L.]) in forest stands on long-term site conditions, drought events, and ozone stress. Trees 25(3): 355-369.

Rouvinen S, T Kuuluvainen. 1997. Structure and asymmetry of tree crowns in relation to local competition in a natural mature Scots pine forest. Canadian Journal for Forest Research 27: 890-902.

SAS (Statistical Analysis System, US). 2004. The SAS System for Windows. Cary, USA. SAS Institute.

Souza AF, C Forgiarini, SJ Longhi, DA Brena. 2008. Regeneration patterns of long-lived dominant conifer and the effects of logging in southern South America. Acta Oecologica 34: 221-232.

Sprugel DG. 1983. Correcting for Bias in Log-Transformed Allometric Equations. Ecology 64: 209-210.

Weiskittel AR, DW Hann, JA Kershaw, JK Vancley. 2011. Forest Growth and Yield Modeling. Chichester, UK. WileyBlackwell. 415 p.

Wykoff WR. 1990. A basal area increment model for individual conifers in northern Rocky Mountains. Forest Science 36(4): 1077-1104.

Zhang L, C Peng, Q Dang. 2004. Individual tree basal area growth models for jack pine and black spruce in northern Ontario. Forestry Chronicle 80(3): 366-374. 